\title{
Pulse propagation of synchrotron radiation through a nuclear two-resonance system
}

\author{
U. van Bürck, ${ }^{1, *}$ W. Potzel, ${ }^{1}$ P. Schindelmann, ${ }^{1}$ Yu. V. Shvyd'ko, ${ }^{2}$ E. Gerdau, ${ }^{2}$ O. Leupold, ${ }^{2,3}$ and H. D. Rüter ${ }^{2}$ \\ ${ }^{1}$ Physik-Department E15, Technische Universität München, D-85748 Garching, Germany \\ ${ }^{2}$ II. Institut für Experimentalphysik, Universität Hamburg, D-22761 Hamburg, Germany \\ ${ }^{3}$ European Synchrotron Radiation Facility, Boîte Postale 220, F-38043 Grenoble, France
}

(Received 9 July 1999; published 8 December 1999)

\begin{abstract}
Pulse propagation through a two-resonance medium was investigated in nuclear forward scattering (NFS) of synchrotron radiation (SR). The SR pulse was coherently transmitted through a target system exhibiting two equivalent resonances, the energy separation of which was varied via the Doppler effect. This allowed a continuous change from a single-resonance medium to a two-resonance system with adjustable splitting. For increasing splitting, the observed time evolutions of NFS changed from a dynamical beat (DB) via hybrid forms of beating into a DB-modulated quantum beat. The experimental results were consistently fitted using a dynamical theory for NFS of SR.
\end{abstract}

PACS number(s): 42.50.Gy, 42.50.Md, 42.25.Bs, 76.80.+y

\section{INTRODUCTION}

The propagation of electromagnetic wave pulses through resonant media [in short, resonant pulse propagation (RPP)] has been of interest since the beginning of this century, when Sommerfeld and Brillouin began to investigate the propagation of a truncated harmonic wave through a strongly dispersive medium [1]. This basic work, which has entered classical textbooks [2], has been largely expanded in the last 20 years (see, e.g., [3], and references therein). Predictions concerning precursor pulses have been experimentally verified in the late 1960s using microwave radiation [4]. The advent of the laser gave a new impetus to theoretical studies of RPP in general (for a review see, e.g., $[5,6]$ ), and in particular of RPP of weak, small-area pulses $[7,8]$. On the experimental side, the picosecond laser technology developed in the 1980 s facilitated broad-band excitation and investigations of RPP of infrared and visible radiation in case of molecular [9], atomic [10], and excitonic [11] resonances. Nowadays, the spread of subpicosecond laser technology implies to an increasing degree the impulsive excitation of resonances and the observation of ultrafast RPP [12].

Studies of RPP at very short wavelengths, however, had been initiated already before, when in the early 1960s the time evolution of Mössbauer $\gamma$ radiation transmitted through resonant absorbers was investigated [13]. In these and later experiments using different pulse shapes (see, e.g., $[14,15]$, and references therein) the rich world of coherent transients was revealed in Mössbauer spectroscopy. However, the low brilliance of the radioactive sources and a high background due to recoil radiation from the source made such experiments difficult. Thus, for the studies of RPP at $\gamma$ wavelengths, great progress was achieved when it became possible to excite the $14.4-\mathrm{keV}$ Mössbauer transition in ${ }^{57} \mathrm{Fe}$ by the short and highly brilliant pulses of synchrotron radiation (SR) [16] and to investigate the time evolution of the practically background-free nuclear forward scattering (NFS) of

*Electronic address: uwe.vbuerck@ph.tum.de
SR [17]. For reviews on nuclear resonance scattering of SR see Refs. [18-20].

Mössbauer transitions are in many ways ideal oscillators, which perfectly embody the concept of a two-level system. They can be strong resonators, corresponding, for instance, in the case of the most common Mössbauer isotope ${ }^{57} \mathrm{Fe}$ to several hundreds of electrons with respect to scattering power. By contrast to the common situation with atomic, molecular, and excitonic resonances, nuclear resonances are isolated in energy. Therefore they can be excited by SR in the so-called impulsive limit, where the bandwidth of the pulse exceeds the natural width of the resonance by a large amount, typically by a factor of $10^{6}$ with nuclear oscillators. Because of the relatively long lifetimes of Mössbauer isotopes, the timescales of resonant scattering are mostly in the easily accessible nanosecond range. In spite of the high brilliance of the present SR sources, RPP of synchrotron $\gamma$ radiation through Mössbauer targets is a single-photon event, corresponding to the propagation of weak, small-area pulses in laser physics [6]. Within this regime, NFS of SR offers attractive possibilities to study RPP. In particular it is easily possible to shift a nuclear resonance by means of the Doppler effect up to hundreds of natural linewidths. This way, simple two-resonance systems with variable energy splitting can be realized, the properties of which with respect to RPP will be studied in the present paper. Such systems can only be achieved for nuclear resonances because of the extreme sharpness in energy of the Mössbauer transitions.

RPP relies on multiple resonant scattering (see, e.g., [2123], and references therein). In a single-resonance medium, multiple scattering proceeds within the same resonance. In this case closed analytical expressions have been derived for the time evolution of RPP at resonant excitation by Mössbauer radiation from a radioactive source [13] and after impulsive excitation by laser radiation and SR [7,8,24,25]. The solutions predicted a characteristic beat with periods increasing with time and decreasing with increasing sample thickness. This beat has been revealed in NFS of SR in singleresonance materials (see [26], and references therein), where it was called dynamical beat (DB). A similar beat has been observed also in RPP of broad-band laser pulses [10-12], 
called, in the case of excitonic resonances, propagation beat or polariton beat.

When the medium exhibits two or more neighboring resonances, inter-resonance interference leads in the singlescattering approximation to a quantum beat (QB) [27]. Interresonance interference is used in atomic [28], molecular [29], excitonic [30], and nuclear [18] QB spectroscopy to determine the separations of resonances. For intensity reasons, however, the targets employed in NFS of SR usually have to be so thick that multiple scattering plays an important role. Multiple scattering via different resonances then seriously complicates the time evolution of NFS. Similar difficulties have been reported for RPP of laser radiation in case of neighboring resonances caused, for example, by Zeeman splitting in an external magnetic field [31] or by a high density of transitions [32].

In the present paper we describe a comprehensive study of RPP through a medium exhibiting two nuclear resonances of equal strength and variable energy separation. For increasing energy separation, beat patterns were observed that changed from a pure DB via complex intermediate forms of beating to a DB-modulated QB. Of fundamental interest here in particular is the influence of a very small resonance splitting, which was found to cause the first minimum of the DB to shift to earlier times.

This effect is also of general importance for the evaluation and interpretation of the time spectra of NFS of SR. The method of NFS of SR is the time-domain coherent-scattering variant of the classical Mössbauer spectroscopy. It is now being used to a rapidly increasing extent at modern second and third generation synchrotron radiation facilities [20]. Because of the coherent nature of the scattering signal, the method exhibits pronounced interference effects that make it highly sensitive to both strength and energy distribution of the nuclear oscillators. Unexpected results of interference in NFS of SR have previously been demonstrated in the case of a continuous asymmetrical distribution of nuclear oscillators [33]. In the present paper such interference effects were studied for the case of two discrete resonances with variable separation.

\section{SAMPLES AND MEASUREMENTS}

Stainless steel (SS) of composition $\mathrm{Fe}_{55} \mathrm{Cr}_{25} \mathrm{Ni}_{20}$, enriched to $95 \%$ in ${ }^{57} \mathrm{Fe}$, was used as sample material. This material is a standard single-line material in Mössbauer spectroscopy, which, however, at room temperature exhibits a slightly broadened resonance linewidth of $\sim 1.6 \Gamma_{0}$, with $\Gamma_{0}$ $\sim 5 \mathrm{neV}$ being the natural linewidth. Foils of thicknesses $\sim 1.3,2.5$, and $7 \mu \mathrm{m}$ were employed. The target consisted of two such SS foils of about equal thickness, mounted downstream behind each other. One foil was fixed; the other foil was moved at constant velocities in the range of $0-20$ $\mathrm{mm} / \mathrm{s}$ by a Mössbauer drive. Special care had been taken to secure the foils in such a way that unwanted vibrations relative to each other were minimized.

The experiments were performed at HASYLAB (Hamburg) at the wiggler beamline BW4. SR of $14.4 \mathrm{keV}$ was monochromatized to 6-meV bandwidth by a high-resolution monochromator $[34,35]$. The SR transmitted through the target was recorded by an avalanche photodiode $[36,37]$. In the five-bunch mode of the storage ring, the time window between the bunches was $\sim 190 \mathrm{~ns}$.

\section{RESULTS AND DISCUSSION}

\section{A. Principle of the method}

In order to study the effect of a variable splitting $\Delta E$ of the two resonances of the target system on the time evolution of NFS, $\Delta E$ was successively enlarged from zero until $\sim 180 \Gamma_{0}$ by increasing the Doppler velocity of one of the foils. The results of two sets of measurements are shown in Figs. 1 and 2. In the first set, the target was composed of two foils of thickness $\sim 1.3 \mu \mathrm{m}$ each. When both foils are at rest, the first minimum of the DB appears at $\sim 100$ ns, i.e., well in the observation window. This set of measurements, which is shown in Fig. 1, focuses mainly on the effect of small splittings $\Delta E$ on the position of the first DB minimum. For the second set of measurements, two foils of thickness $\sim 7 \mu \mathrm{m}$ each were chosen. Now the first three DB minima fall into the observation window when both foils are at rest. This set of measurements, which is displayed in Fig. 2, mainly illuminates the blending of DB and QB in the transition from zero to very large resonance splittings.

Such target systems composed of two separate foils $[38,22]$ have, for instance, been employed in NFS of SR as an interferometer in time and energy space. For this application, they have been used either at zero energy splitting [39], or at very large splitting [40]. In both cases a change or loss of the phasing between the reemitted wave packets and, for zero splitting, a change or loss of the radiative coupling between both target foils is measured. This way perturbations of one of the resonant foils $[39,41-45]$ or of an intermediate nonresonant scatterer $[40,46,47]$ have been investigated.

For the interpretation of the present study it is important to note that, with respect to NFS, the employed target system of two separate foils is equivalent to a two-resonance medium. This is true as long as the polarization is not changed. The equivalence relies on NFS being a spatially coherent scattering process. Analytically this equivalence is based on the additivity of coherent nuclear resonance scattering amplitudes of different resonances (see, e.g., [48]), which allows us to write the refractive index as unity plus the sum of the contributions caused by different nuclear resonances [49].

\section{B. Dynamical beat and DB-modulated quantum beat}

In the case of a single nuclear resonance, multiple scattering leads to a characteristic modulation of the NFS radiation amplitude $A(t)$ at time $t$ after an instantaneous excitation of a nuclear ensemble at time $t=0$ [25],

$$
A(t) \propto T e^{-i \omega_{0} t-\tau / 2} \sigma(T \tau) .
$$

Here $\omega_{0}$ is the nuclear resonance frequency, $\sigma(x)$ $=J_{1}(\sqrt{x}) / \sqrt{x}$ where $J_{1}$ is the Bessel function of first kind and first order, $\tau=t / \tau_{0}$ is the time normalized with respect to the nuclear lifetime $\tau_{0}$, and $T=\sigma_{0} N d f_{L M}$ is the effective 

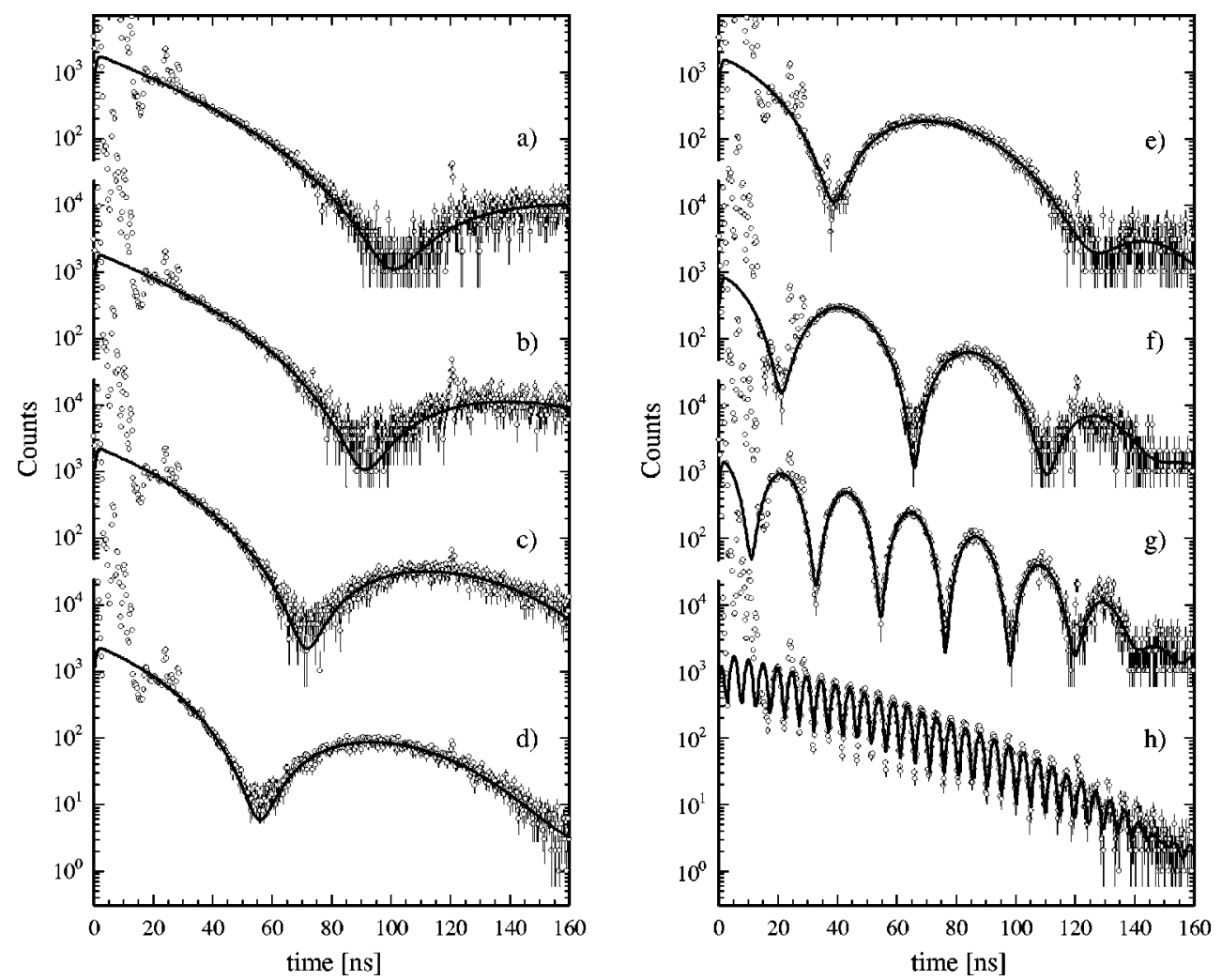

FIG. 1. Time evolution of NFS of SR from two $\sim 1.3-\mu \mathrm{m}$ SS foils, where one foil was moved at constant velocities corresponding to Doppler shifts of 0 (a), 2 (b), 4 (c), 6 (d), 10 (e), 20 (f), 40 (g), and $180 \Gamma_{0}$ (h). The small spikes at $\sim 120$ ns are due to spurious bunches. The solid lines are fits using the NFS theory [23] with effective thickness $T=2 T^{\prime}=19$.

(Mössbauer) thickness of the sample, with $\sigma_{0}$ being the nuclear resonance cross section, $N$ the density of resonant nuclei per unit volume, $d$ the sample thickness, and $f_{L M}$ the Lamb-Mössbauer factor. The resulting modulation of the NFS intensity is the DB. Equivalent expressions for RPP of laser radiation in the impulsive limit have been derived in $[7,8,24]$. The characteristic beat of $A(t)$ expressed by Eq. (1) has been observed in RPP of electromagnetic radiation in a very wide range of wavelengths and for resonances of quite different nature (for a review see [26]).

An explanation of the DB based on the picture of interference in energy domain was obtained in Ref. [19] by considering the energy spectrum of the NFS intensity $|A(E)|^{2}$, where $A(E)=1-R(E)$ with $R(E)$ the transmission amplitude $[25,19]$. Such a spectrum is displayed in Fig. 3(a) for a single-resonance material. The spectrum exhibits a doublehump structure that is typical for optically thick samples [50], and that arises from enhanced scattering and diminished absorption on the wings of the resonance. In this picture, the DB results from the interference of the dominant contributions above and below resonance (intraresonance interference). These contributions with separation $\simeq \hbar \omega_{D}$ lead to a DB with frequency of order $\omega_{D}$, which is also displayed in Fig. 3(a).

In the case of several resonances, multiple scattering can proceed also via different resonances, leading in general to complicated time responses discussed in Secs. III C and III D. But obviously multiple scattering via different resonances can be neglected when the energy separations of the resonances are very large in comparison with the extensions of their respective double-hump structures. In this case, one can make the approximation (see, e.g., $[15,33]$ ) that the time evolution of the amplitudes $A_{l}(t)$ of the radiation components of resonance $l$ are approximately given by Eq. (1), where for each resonance of energy $\hbar \omega_{l}$ the appropriate effective thickness $T_{l}$ is used. The resulting NFS amplitude will then be given by the sum of all contributions:

$$
A(t) \propto \sum_{l} A_{l}(t)=\sum_{l} T_{l} e^{-i \omega_{l} t-\tau / 2} \sigma\left(T_{l} \tau\right) .
$$

In the case of two resonances with equal effective thickness $T_{l}=T^{\prime}$ this approximation leads to a NFS intensity given by

$$
I(t) \propto T^{\prime 2} \cos ^{2}\left(\frac{\omega_{Q} t}{2}\right) e^{-\tau} \sigma^{2}\left(T^{\prime} \tau\right)
$$

with $\omega_{Q}=\omega_{1}-\omega_{2}$. The $\cos ^{2}$ term describes the periodic modulation of the signal due to inter-resonance interference, called QB $[27,51,49]$. The $\sigma^{2}$ term describes the aperiodic DB modulation due to multiple scattering within each resonance. Since the effects of inter-resonance and intraresonance interference are factorized, Eq. (3) describes the superposition of a fast $\mathrm{QB}$ and a slow $\mathrm{DB}$ envelope, which we shall call a DB-modulated QB. A typical NFS energy spectrum calculated for the case of two well-separated transitions of equal strengths is depicted in Fig. 3(b). The corresponding 

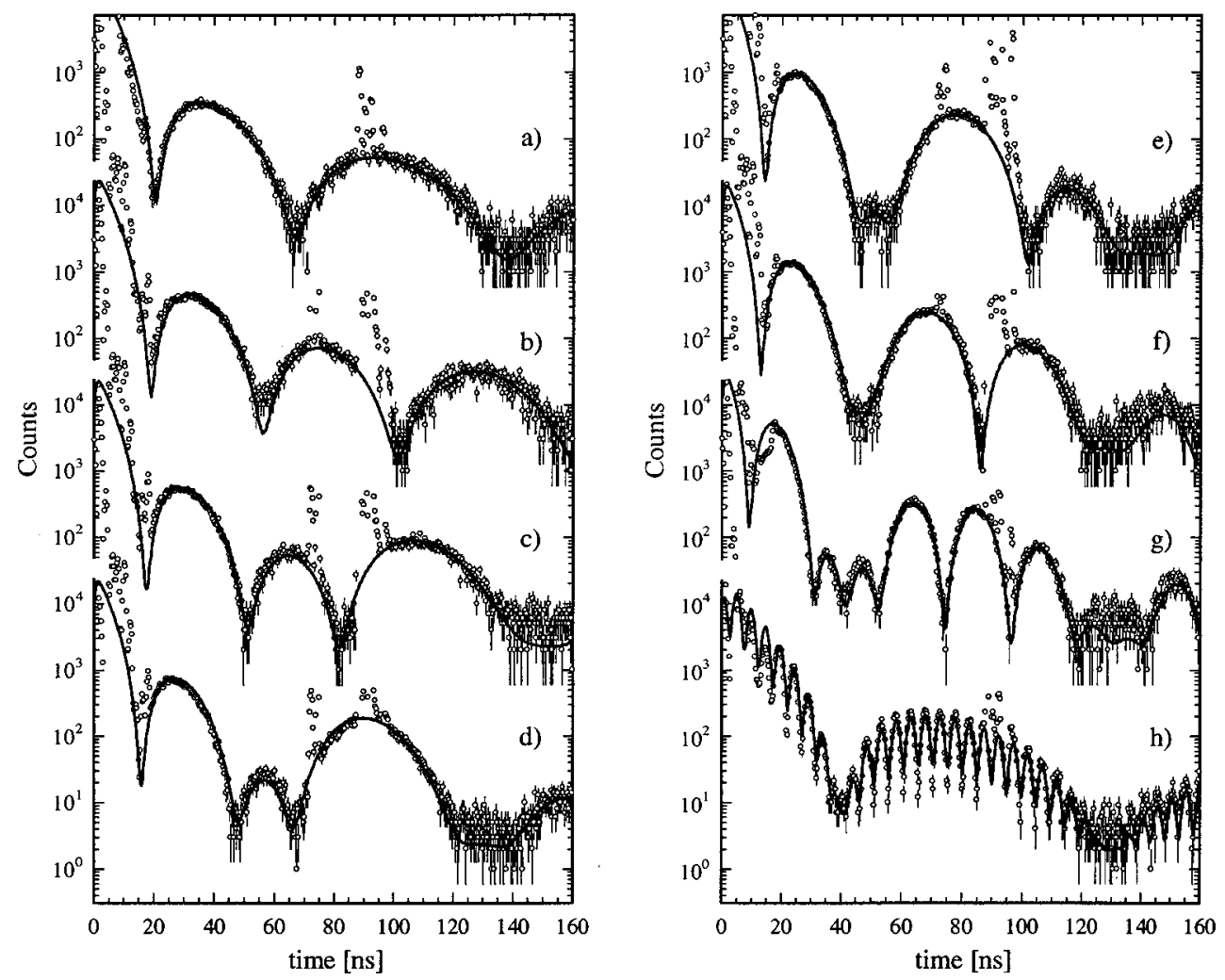

FIG. 2. Time evolution of NFS of SR from two $\sim 7-\mu \mathrm{m}$ SS foils, where one foil was moved at constant velocities corresponding to Doppler shifts of 0 (a), 8 (b), 12 (c), 16 (d), 20 (e), 24 (f), 40 (g), and 180 $\Gamma_{0}(\mathrm{~h})$. The spikes centered at $\sim 72$ and 92 ns are due to spurious bunches. The solid lines are fits using the NFS theory [23] with effective thickness $T=2 T^{\prime}=104$.

time spectrum exhibits a DB-modulated QB. Note that due to the splitting of the single resonance into two resonances, the effective thickness of each of the separated resonances is decreased by a factor of 2 , leading to a twice-smaller separation $\hbar \omega_{D}^{\prime}$ of the double humps and to a twice-slower DBmodulation of the QB as compared with the DB of Fig. 3(a).

In reality, however, there remains also in the case of farseparated resonances a weak influence of multiple scattering via different resonances. This effect can be taken into account in Eq. (3) in first approximation by an additional phase in the argument of the $\cos ^{2}$ term, which describes a slight shift of the QB pattern to earlier times proportional to sample thickness [49]. In the double-hump picture of Fig. 3(b) this remaining influence is noticed by the slight asymmetry of both double humps.

In the present experiment, the measurements with the two foils at rest $(\Delta E=0)$ shown in Figs. 1(a) and 2(a) represent good examples for a DB as described by Eq. (1). In this case both foils have the same resonance energy. Therefore all nuclei are coupled via single-resonance multiple scattering of the radiation field, and the DB is determined by the total thickness of the two foils.

The measurements at very large splitting $\left(\Delta E=180 \Gamma_{0}\right)$ shown in Figs. 1(h) and 2(h), by contrast, represent examples for the opposite limit described by Eq. (3). Now the nuclei of the two foils have strongly different resonance energies, and multiple scattering proceeds for each resonance separately. Thus, only the nuclei within each foil are coupled via multiple scattering of the radiation field. This leads to a separate dynamical modulation of each of the radiation fields emitted by the two foils. In addition, the interference of these two wave packets yields a QB. The time evolutions shown in Figs. 1(h) and 2(h) clearly exhibit DB modulations, which are twice as slow as those observed at $\Delta E=0$, and which are superimposed on a QB with $\sim 5$-ns period corresponding to $\Delta E=180 \Gamma_{0}$.

Already a splitting of $\Delta E=40 \Gamma_{0}$ [Figs. $1(\mathrm{~g})$ and 2(g)] seems to be large enough to allow an interpretation of the observed beat as a DB-modulated QB. The fast beat exhibits an almost regular pattern with period of $\sim 22 \mathrm{~ns}$, corresponding to the actual resonance splitting. And the slow envelopes are practically identical to those observed for $\Delta E$ $=180 \Gamma_{0}$. Note that DB envelope and QB are strictly superimposed and do not interfere. This leads, for instance, to the remarkable sharp cut of the QB at $\sim 40 \mathrm{~ns}$ in Fig. 2(g) that does not appear in the corresponding time evolution for the thinner target [Fig. 1(g)]. For the thicker target, both wave packets reemitted from the two foils have the first DB minimum at $\sim 40 \mathrm{~ns}$, and this vanishing factor suppresses the QB interference pattern at this instant in Fig. 2(g).

\section{Shift of the first DB minimum to earlier times}

The effect of a small resonance splitting on RPP is not obvious. As seen above, a large splitting leads to a reduction of the effective thickness in each resonance by a factor of 2 and thus to twice longer periods of the DB. From this result one could expect an effect into the same direction also for a 


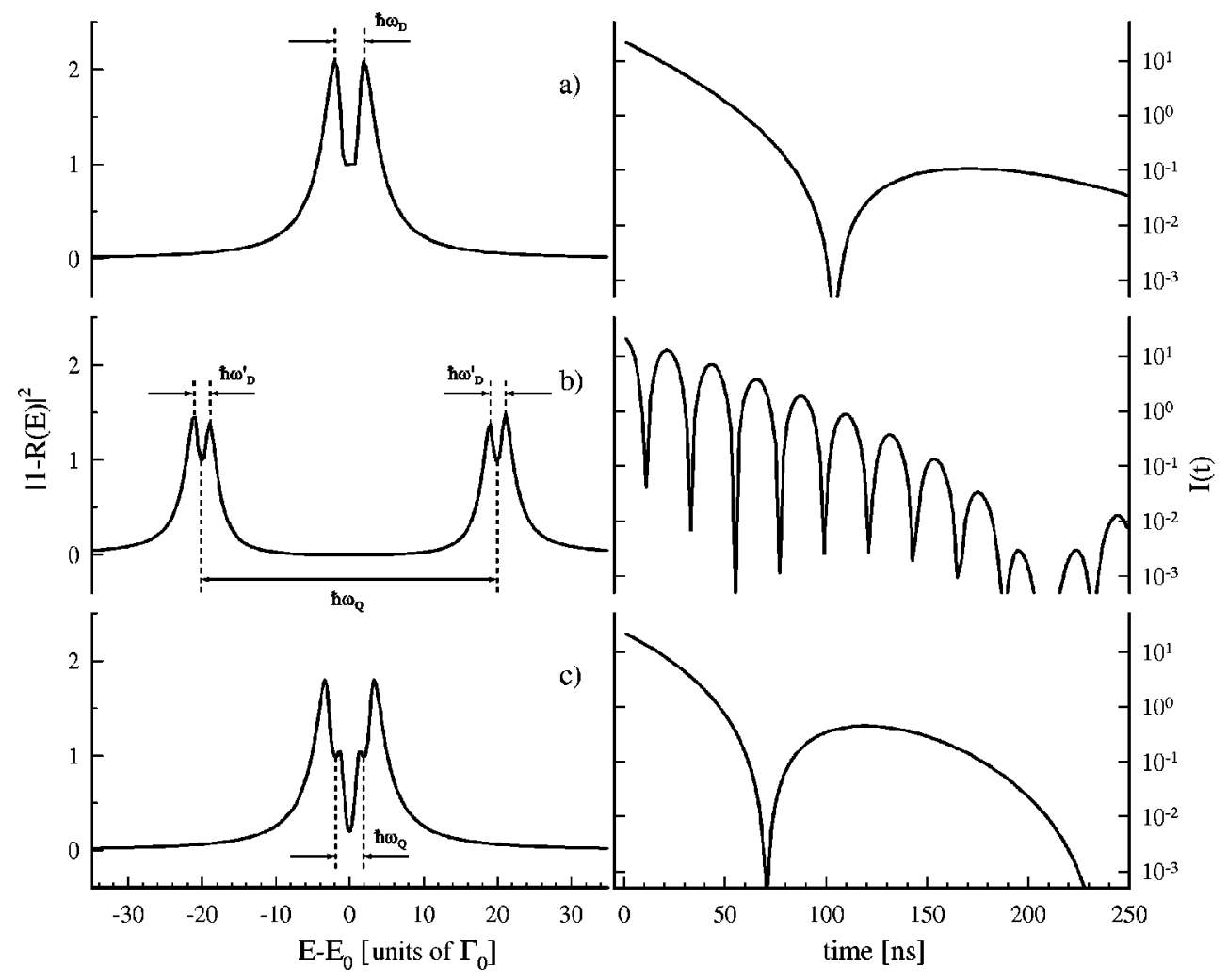

FIG. 3. Energy and time dependences of the NFS intensity in case of an optically thick scatterer $(T=20)$ with one resonance (a) and two resonances of natural linewidth separated by a large $\left(\hbar \omega_{Q}=40 \Gamma_{0}\right)(\mathrm{b})$ or a small $\left(\hbar \omega_{Q}=4 \Gamma_{0}\right)$ (c) splitting. Each resonance shows the typical double-hump structure, with separation of the two humps by $\hbar \omega_{D}$ [in (a)] and $\hbar \omega_{D}^{\prime}$ [in (b)]. Note that $\omega_{D}^{\prime} \simeq \omega_{D} / 2$. Situation (a) yields a DB with apparent frequency of order $\omega_{\mathrm{D}}$. Situation (b) gives a time evolution characterized by a fast QB with frequency $\omega_{Q}$, modulated by a DB with a frequency of order $\omega_{D}^{\prime}$. Situation (c) leads to a shift of the DB minimum to earlier times.

small splitting, yielding a shift of the first DB minimum to later times. On the other hand, due to the onset of destructive interference of two wave packets centered at slightly different energies, the evolution of the phase should lead to an additional decay of the NFS signal and thus to a shift of the first DB minimum to earlier times. It is not clear which one of these two opposite effects will dominate. The cases of Lorentzian and Gaussian resonance broadening, for instance, which are not so much different from small resonance splittings, predict for the first DB minimum no shift or a shift towards earlier times, respectively [33].

The experimental answer is unambiguous: The DB minimum is immediately shifted to earlier times. Already when a small splitting of $\sim 2 \Gamma_{0}$ is introduced [Fig. 1(b)], the DB minimum is shifted from $\sim 100$ to $92 \mathrm{~ns}$. This shift to earlier times goes on continuously for increasing splitting: $\Delta E=4$ or $6 \Gamma_{0}$ yields a shift of the first beat minimum to $\sim 71$ or 56 ns [Figs. 1(c,d)], respectively. This shift to earlier times demonstrates that the phase evolution due to inter-resonance interference dominates over the reduction of the effective thickness.

For larger values of the splitting, the time evolutions of NFS approach more and more the shape of a DB-modulated QB. The slow DB envelope with a first minimum at $\sim 200 \mathrm{~ns}$ outside the observation window becomes evident for $\Delta E$ $\geqslant 20 \Gamma_{0}$ [Fig. 1(f)]. In parallel, the observed fast beats correspond to an increasing degree in shape and period to a $\mathrm{QB}$, which should exhibit periods of $\sim 88,44$, and $22 \mathrm{~ns}$ for splittings of 10,20 , and $40 \Gamma_{0}$, respectively [compare Figs. $1(\mathrm{e}, \mathrm{f}, \mathrm{g})]$. As discussed in Sec. III B, the beat pattern observed at $\Delta E=40 \Gamma_{0}$ can already be considered in good approximation as a DB-modulated QB.

In NFS by two foils of different resonance energies $\hbar \omega_{a}$ and $\hbar \omega_{b}$ and of different effective thicknesses $T_{a}$ and $T_{b}$ the radiation amplitude can be written as [22]

$$
A_{a b}(t) \propto e^{-i \omega_{b} t-\tau / 2} B_{a b}(t)
$$

with a slowly varying envelope given by

$$
\begin{aligned}
B_{a b}(t)= & T_{a} \sigma\left(T_{a} \tau\right) e^{-i\left(\omega_{a}-\omega_{b}\right) t}+T_{b} \sigma\left(T_{b} \tau\right) \\
& -\frac{T_{a} T_{b}}{2 \tau_{0}} \int_{0}^{t} d t^{\prime} e^{-i\left(\omega_{a}-\omega_{b}\right) t^{\prime}} \sigma\left(T_{a} \tau^{\prime}\right) \sigma\left[T_{b}\left(\tau-\tau^{\prime}\right)\right],
\end{aligned}
$$

where $\tau^{\prime}=t^{\prime} / \tau_{0}$. For $\Delta E=\hbar\left(\omega_{a}-\omega_{b}\right)=0$ this expression is equivalent to

$$
B_{a b}(t)=\left(T_{a}+T_{b}\right) \sigma\left[\left(T_{a}+T_{b}\right) \tau\right] .
$$

Equation (6) describes a pure DB where the envelope $B_{a b}(t)$ is real. This also causes the intensity at the first DB minimum to vanish completely. For small splittings $\Delta E$ and 
short times $t$ the complex envelope $B_{a b}(t)$ given by Eq. (5) can be expanded in terms of $\left(\omega_{a}-\omega_{b}\right) t$ and $\left(\omega_{a}-\omega_{b}\right) t^{\prime}$. The expansion shows that for small $\Delta E \neq 0$ the intensity at the former position of the first $\mathrm{DB}$ is proportional to $\left(\omega_{a}\right.$ $\left.-\omega_{b}\right)^{2}$ and does not reach zero any more. For a further analysis, the function $\sigma(x)$ must be expanded in order to evaluate the integral in Eq. (5). We find, however, that such an expansion does not contribute to elucidate the problem of the shift of the first DB minimum.

Instead, more insight can be gained from the doublehump picture [19]. The situation with two closely lying resonances is depicted in Fig. 3(c). Here we can see that a resonance splitting, which is small in comparison with or of the same order of magnitude as the separation of the two humps of a double hump, leads to an increase of the effective double-hump separation. An increased double-hump splitting, however, means a faster beating and thus a shift of the beat minima to earlier times. The point is why the double hump is changed in that specific way. The reason is found in the interference of the wave packets from neighboring oscillators, like in the case of an asymmetric continuous oscillator distribution [33]. The pronounced dispersion around each of the resonances causes destructive interference of the forward-scattering amplitudes in the region in between (i.e., above the lower and below the higher resonance) and constructive interference in the region outside of the split resonances. This leads to an effective increase of the doublehump separation.

When we follow the fate of the first minimum of the beat, we notice a continuous shift of its position, starting from $\sim 100 \mathrm{~ns}$ for $\Delta E=0$ [Fig. 1(a)] and reaching $\sim 11 \mathrm{~ns}$ for $\Delta E=40 \Gamma_{0}$ [Fig. $\left.1(\mathrm{~g})\right]$. In parallel its character is changed completely. It starts at $\Delta E=0$ as a pure $\mathrm{DB}$ minimum, caused by multiple scattering within one resonance. And it ends up for large values of $\Delta E$ as a $\mathrm{QB}$ minimum, caused by the interference of wave packets of different energies. The modulation patterns observed in the intermediate region have to be interpreted as the result of an entangled combination of intraresonance and inter-resonance interference.

These model experiments are also of practical importance for the evaluation of NFS spectra. They give a warning, that a shift of the DB minimum to earlier times could not only be caused as usual by an increase of the effective thickness (e.g., due to an increase of the Lamb-Mössbauer factor $f_{L M}$ ), but also by a small splitting in energy [52]. In studies of the temperature dependence of the Lamb-Mössbauer factor, a shift of the DB minimum to earlier times could therefore easily be misinterpreted. The ambiguity can only be avoided by choosing the experimental time window sufficiently large to cover also the second DB minimum. Often, however, the effective thickness of the sample is so small that even the first DB minimum is outside of the observation window. Such conditions are the rule for biological or other diluted samples. Then only the time evolution of the initial decay of the NFS signal is observed, which is determined not only by the effective thickness, but possibly also by relaxation [53] or diffusion [54,55] effects or just by a small hyperfine splitting. A unique interpretation is then difficult if no additional information is available.

\section{Hybrid beat for the thicker target}

The blending of DB and QB into hybrid forms of beating was also studied in a different thickness regime, where several dynamical beats fall into the observation window. By contrast to the previous view, which was centered mainly on the fate of the first DB minimum under the influence of a small resonance splitting, the present case allows us to follow the hybridization of the first three DB minima (see Fig. 2).

In particular, we studied the time evolution of NFS for resonance splittings $\Delta E$ in the range from 8 to $24 \Gamma_{0}$ [Figs. $2(\mathrm{~b}-\mathrm{f})]$. In this range, the $\mathrm{QB}$ period of inter-resonance interference would vary from $\sim 111 \mathrm{~ns}$ at $\Delta E=8 \Gamma_{0}$ to $\sim 37 \mathrm{~ns}$ at $\Delta E=24 \Gamma_{0}$. This means $\mathrm{QB}$ and DB would exhibit comparable periods, and therefore strong effects of hybridization can be expected. Below and above this transition regime, i.e., at $\Delta E=0$ and $\Delta E \geqslant 40 \Gamma_{0}$, the beat can be identified as a DB and a DB-modulated $\mathrm{QB}$, respectively.

For increasing $\Delta E$, the first minimum of the beat is again continuously shifted to earlier times, starting at $\sim 20 \mathrm{~ns}$ for $\Delta E=0$ [Fig. 2(a)] and reaching $\sim 11 \mathrm{~ns}$ for $\Delta E=40 \Gamma_{0}$ [Fig. $2(\mathrm{~g})]$. Thus, like for the thin target, the first DB minimum continuously goes over into a first QB minimum. From this behavior one could expect also for the remaining beats a similar development for increasing $\Delta E$ : shift to earlier times and compression, until the beats correspond to the respective QB. Such a simple compression, however, is not observed. The transition from DB to DB-modulated QB is more complicated, also including, for instance, the annihilation of a beat. Such an annihilation is observed for the third beat with maximum at $\sim 100 \mathrm{~ns}$ in the time evolution of the DB [Fig. 2(a)]. For increasing $\Delta E$, this beat is shifted to earlier times, with both width and intensity continuously decreasing [Figs. 2(b-e)]. It finally disappears for $\Delta E=24 \Gamma_{0}$ at $\sim 47 \mathrm{~ns}$ [Fig. 2(f)]. Altogether the intermediate steps in the transition from DB to DB-modulated QB are characterized by complex beat patterns, which again represent hybrid forms of beating.

Nevertheless, these sets of quite different beat patterns observed in case of the thinner (Fig. 1) and of the thicker target (Fig. 2) have been consistently fitted using the NFS theory [23]. In these fits, the slightly broadened resonance linewidth of SS at room temperature of $\sim 1.6 \Gamma_{0}$ was modeled by a small hyperfine splitting corresponding to a random magnetic field of $0.589 \mathrm{~T}$. This model takes into account small deviations at late times of the time evolutions of Figs. 1 and 2 from those for a target of natural linewidth. For each set of measurements, the foil thicknesses were kept fixed, with slightly different values of 1.1 and 1.5 (6.7 and 7.3) $\mu \mathrm{m}$ for the foils of each pair. The values of the parameter $\Delta E$ were those obtained previously from a calibration of the constant velocity drive. The good agreement between experiment and theory demonstrates that the time evolution of RPP in a two-resonance medium can be fully described by theory in case of NFS of SR.

\section{SUMMARY}

RPP in a two-resonance medium is made complicated by multiple scattering via different resonances. Analytical solu- 
tions exist only in the limiting cases of no splitting or of very large splitting, where multiple scattering via different resonances can practically be neglected. For impulsive excitation, these solutions yield in the first case a DB, and in the second case a superposition of a DB envelope over a QB. For the transition region in between these limits, numerical solutions have to be employed.

This transition region of RPP was investigated in the present study in the regime of $\gamma$ radiation with $\sim 1$ - $\AA$ wavelength. A target system consisting of two foils was used to continuously vary the resonance splitting via the Doppler effect. Sets of measurements of NFS of SR were performed for targets of different thicknesses. For the limiting cases of zero and very large splitting, beat patterns were observed that can be easily interpreted as DB and DB-modulated QB, respectively. For the transition in between, hybrid forms of beating with generally complex beat patterns were found.

Already a small resonance splitting made the first minimum of the DB shift to earlier times. This exhibits the influence of inter-resonance interference on multiple scattering.
In studies of the temperature dependence of Mössbauer parameters via NFS of SR, such shifts could be easily misinterpreted when the observation window is not large enough.

The sets of measurements were consistently fitted using a dynamical theory for NFS. We conclude that the time evolution of RPP in a medium exhibiting two equivalent resonances of arbitrary splitting can be fully described in the case of NFS of SR.

The present paper focuses on RPP in the case of two identical resonances with variable separation in energy. The interesting case of two separate resonances of different widths is presently under study [56].

\section{ACKNOWLEDGMENTS}

This work has been funded by the Bundesministerium für Bildung, Wissenschaft, Forschung und Technologie under Contracts No. 05 643WOA/SK8WOA and No. 05 643GUA1. The authors are grateful to G.V. Smirnov and V.G. Kohn for fruitful discussions.
[1] A. Sommerfeld, Ann. Phys. (Leipzig) 44, 177 (1914); L. Brillouin, ibid. 44, 203 (1914); Wave Propagation and Group Velocity (Academic Press, New York, 1960).

[2] J.A. Stratton, Electromagnetic Theory (McGraw-Hill, New York, 1941), Sec. 5.18; J.D. Jackson, Classical Electrodynamics, 2nd ed. (Wiley, New York, 1975), Sec. 7.11.

[3] K.E. Oughstun and C.M. Balictsis, Phys. Rev. Lett. 77, 2210 (1996).

[4] P. Pleshko and I. Palócz, Phys. Rev. Lett. 22, 1201 (1969).

[5] G.L. Lamb, Jr., Rev. Mod. Phys. 43, 99 (1971).

[6] L. Allen and J.H. Eberly, Optical Resonance and Two-Level Atoms (Wiley, New York, 1975).

[7] D.C. Burnham and R.Y. Chiao, Phys. Rev. 188, 667 (1969).

[8] M.D. Crisp, Phys. Rev. A 1, 1604 (1970).

[9] H.-J. Hartmann and A. Laubereau, Opt. Commun. 47, 117 (1983).

[10] J.E. Rothenberg, D. Grischkowsky, and A.C. Balant, Phys. Rev. Lett. 53, 552 (1984).

[11] D. Fröhlich, A. Kulik, B. Uebbing, A. Mysyrowicz, V. Langer, H. Stolz, and W. von der Osten, Phys. Rev. Lett. 67, 2343 (1991).

[12] M. Matusovsky, B. Vaynberg, and M. Rosenbluh, J. Opt. Soc. Am. B 13, 1994 (1996).

[13] F.J. Lynch, R.E. Holland, and M. Hamermesh, Phys. Rev. 120, 513 (1960).

[14] E. Ikonen, P. Helistö, T. Katila, and K. Riski, Phys. Rev. A 32, 2298 (1985); I. Tittonen, M. Lippmaa, P. Helistö, and T. Katila, Phys. Rev. B 47, 7840 (1993).

[15] Yu.V. Shvyd'ko, S.L. Popov, and G.V. Smirnov, J. Phys.: Condens. Matter 5, 1557 (1993); 5, 7047(E) (1993); Yu.V. Shvyd'ko, G.V. Smirnov, S.L. Popov, and T. Hertrich, Pis'ma Zh. Éksp. Teor. Fiz. 53, 69 (1991) [JETP Lett. 53, 69 (1991)].

[16] E. Gerdau, R. Rüffer, H. Winkler, W. Tolksdorf, C.P. Klages, and J.P. Hannon, Phys. Rev. Lett. 54, 835 (1985).

[17] J.B. Hastings, D.P. Siddons, U. van Bürck, R. Hollatz, and U.
Bergmann, Phys. Rev. Lett. 66, 770 (1991).

[18] E. Gerdau and U. van Bürck, in Resonant Anomalous X-Ray Scattering, edited by G. Materlik, C.J. Sparks, and K. Fischer (North-Holland, Amsterdam, 1994), p. 589.

[19] G.V. Smirnov, Hyperfine Interact. 97/98, 551 (1996); in X-Ray and Inner-Shell Processes, edited by R.L. Johnson, H. Schmidt-Böcking, and B.F. Sonntag, AIP Conf. Proc. No. 389 (AIP, New York, 1997), p. 323.

[20] Nuclear Resonant Scattering of Synchrotron Radiation, edited by E. Gerdau and H. de Waard (Baltzer, Oxford, in press).

[21] R.W. James, The Optical Principles of the Diffraction of X-Rays (G. Bell and Sons, London, 1958).

[22] G.V. Smirnov, in Nuclear Resonant Scattering of Synchrotron Radiation (Ref. [20]).

[23] Yu.V. Shvyd'ko, Phys. Rev. B 59, 9132 (1999).

[24] A. Laubereau and W. Kaiser, Rev. Mod. Phys. 50, 607 (1978).

[25] Yu. Kagan, A.M. Afanas'ev, and V.G. Kohn, J. Phys. C 12, 615 (1979).

[26] U. van Bürck, in Nuclear Resonant Scattering of Synchrotron Radiation (Ref. [20]).

[27] G.T. Trammell and J.P. Hannon, Phys. Rev. B 18, 165 (1978); 19, 3835(E) (1979).

[28] S. Haroche, in High-Resolution Laser Spectroscopy, edited by K. Shimoda (Springer, Berlin, 1976), p. 253.

[29] H.-J. Hartmann and A. Laubereau, J. Chem. Phys. 80, 4663 (1984).

[30] W. von der Osten, V. Langer, and H. Stolz, in Coherent Optical Interactions in Semiconductors, edited by R.T. Phillips (Plenum Press, New York, 1994), p. 111; K.-H. Pantke and J.M. Hvam, Int. J. Mod. Phys. B 8, 73 (1994).

[31] D. Fröhlich, A. Kulik, B. Uebbing, V. Langer, H. Stolz, and W. von der Osten, Phys. Status Solidi B 173, 31 (1992).

[32] U. Neukirch, K. Wundke, J. Gutowski, and D. Hommel, Phys. Status Solidi B 196, 473 (1996).

[33] Yu.V. Shvyd'ko, U. van Bürck, W. Potzel, P. Schindelmann, 
E. Gerdau, O. Leupold, J. Metge, H.D. Rüter, and G.V. Smirnov, Phys. Rev. B 57, 3552 (1998).

[34] D.P. Siddons, J.B. Hastings, and G. Faigel, Nucl. Instrum. Methods Phys. Res. A 266, 329 (1988).

[35] In the experiment a high-resolution monochromator was employed with channel-cut $\operatorname{Si}\left(\begin{array}{lll}4 & 2 & 2\end{array}\right)$ and $\operatorname{Si}\left(\begin{array}{lll}1 & 2 & 2\end{array}\right)$ crystals in a nested geometry, designed by E. Gerdau, R. Rüffer, and H.D. Rüter on the basis of a proposal by T. Ishikawa et al., Rev. Sci. Instrum. 63, 1015 (1992).

[36] A.Q.R. Baron, Nucl. Instrum. Methods Phys. Res. A 352, 665 (1995); A.Q.R. Baron, R. Rüffer, and J. Metge, ibid. 400, 124 (1997).

[37] S. Kishimoto, J. Synchrotron Radiat. 5, 275 (1998).

[38] S. Kikuta, Y. Yoda, Y. Hasegawa, K. Izumi, T. Ishikawa, X.W. Zhang, S. Kishimoto, H. Sugiyama, T. Matsushita, M. Ando, C.K. Suzuki, M. Seto, H. Ohno, and H. Takei, Hyperfine Interact. 71, 1491 (1992).

[39] G.V. Smirnov, U. van Bürck, J. Arthur, S.L. Popov, A.Q.R. Baron, A.I. Chumakov, S.L. Ruby, W. Potzel, and G.S. Brown, Phys. Rev. Lett. 77, 183 (1996).

[40] A.Q.R. Baron, H. Franz, A. Meyer, R. Rüffer, A.I. Chumakov, E. Burkel, and W. Petry, Phys. Rev. Lett. 79, 2823 (1997).

[41] U. van Bürck, W. Potzel, P. Schindelmann, G.V. Smirnov, S.L. Popov, E. Gerdau, O. Leupold, Yu.V. Shvyd'ko, and H.D. Rüter, HASYLAB Annual Report 1996 (unpublished), p. 888.

[42] G.V. Smirnov, S.L. Popov, U. van Bürck, W. Potzel, P. Schindelmann, E. Gerdau, O. Leupold, Yu.V. Shvyd'ko, and H.D. Rüter, HASYLAB Annual Report 1996 (unpublished), p. 890.

[43] U. van Bürck, G.V. Smirnov, and J. Arthur, ESRF Experimental Report No. HS-122, 1997 (unpublished).

[44] P. Schindelmann, W. Potzel, U. van Bürck, G.V. Smirnov, S.L. Popov, E. Gerdau, Yu.V. Shvyd'ko, and H.D. Rüter, HASYLAB Annual Report 1998 (unpublished), p. 951; U. van
Bürck and G.V. Smirnov, ESRF Experimental Report No. MI273, 1999 (unpublished).

[45] G.V. Smirnov and W. Potzel, in Nuclear Resonant Scattering of Synchrotron Radiation (Ref. [20]).

[46] H. Jex, A. Ludwig, F.J. Hartmann, E. Gerdau, and O. Leupold, Europhys. Lett. 40, 317 (1997).

[47] A. Ludwig and H. Jex, Physica B 254, 1 (1998).

[48] Yu. Kagan and A.M. Afanas'ev, Z. Naturforsch. A 28, 1351 (1973).

[49] U. van Bürck, D.P. Siddons, J.B. Hastings, U. Bergmann, and R. Hollatz, Phys. Rev. B 46, 6207 (1992).

[50] At present, such spectra cannot be measured in case of NFS of SR. Comparable spectra for the propagation of laser pulses were recently reported by J.K. Ranka, R.W. Schirmer, and A.L. Gaeta, Phys. Rev. A 57, R36 (1998).

[51] E. Gerdau, R. Rüffer, R. Hollatz, and J.P. Hannon, Phys. Rev. Lett. 57, 1141 (1986).

[52] In the given time window, the time evolution for $\Delta E=2 \Gamma_{0}$ [Fig. 1(b)] for instance could equally well be fitted assuming $\Delta E=0$ and allowing for a $10 \%$ larger optical thickness instead. Similar ambiguities for larger energy splittings were recently pointed out by M. Haas, E. Realo, H. Winkler, W. Meyer-Klaucke, A.X. Trautwein, O. Leupold, and H.D. Rüter, Phys. Rev. B 56, 14082 (1997).

[53] A. Meyer, H. Franz, J. Wuttke, W. Petry, N. Wiele, R. Rüffer, and C. Hübsch, Z. Phys. B: Condens. Matter 103, 479 (1997).

[54] B. Sepiol, A. Meyer, G. Vogl, H. Franz, and R. Rüffer, Phys. Rev. B 57, 10433 (1998).

[55] G.V. Smirnov and V.G. Kohn, Phys. Rev. B 52, 3356 (1995); V.G. Kohn and G.V. Smirnov, ibid. 57, 5788 (1998).

[56] W. Potzel, U. van Bürck, P. Schindelmann, G.V. Smirnov, E. Gerdau, Yu.V. Shvyd'ko, and H.D. Rüter, HASYLAB Annual Report 1998 (unpublished), p. 953. 